\title{
Field distribution in a coaxial electrostatic wiggler
}

\author{
Shi-Chang Zhang* \\ Institute of Photoelectronics, Campus Mail Box 50, School of Information Science and Technology, \\ Southwest Jiaotong University, Chengdu, Sichuan 610031, People's Republic of China
}

(Received 19 March 2010; published 24 September 2010)

\begin{abstract}
The field distribution in a coaxial electrostatic wiggler corresponds to the special solution of a Laplace equation in a cylindrical coordinate system with a boundary value problem of sinusoidal ripples. This paper is devoted to the physical and mathematical treatment for an analytical solution of the field distribution in the coaxial electrostatic wiggler. The explicit expression of the solution indicates that the field distribution in the coaxial electrostatic wiggler varies according to a periodic function in the longitudinal direction, and is related to the first and second kinds of modified Bessel functions in the radial direction, respectively. Comparison shows excellent agreement between the analytical formula and the computer simulation technology (CST) results. The physical application of the considered system and its analytical solution are discussed.
\end{abstract}

DOI: 10.1103/PhysRevSTAB.13.090701

PACS numbers: 41.20.Cv, 42.25.Fx, 02.30.Jr

\section{INTRODUCTION}

A free-electron laser (FEL) based on the three-wave (incident wave, pump wave, and space-charge wave) interaction mechanism in an electrostatic wiggler for a relativistic electron beam to generate millimeter waves was first suggested by Bekefi and Shefer [1,2]. Then it was verified by in-principle experiments [3,4], and further studies were continued by several authors [5-11]. Typically, the electrostatic wiggler is a configuration with the periodic electrostatic field distribution produced by the ripple corrugations on the inside surface of the metallic pipe connected with an electrostatic voltage [1,2]. Without loss of generality, it can be represented by the structure shown in Fig. 1, where the outside surface of the inner rod with a radius $r_{\text {in }}$ is smooth and grounded and the inside surface of the outer conductor is connected to a negative voltage $-V_{0}$ and sinusoidally corrugated with a mean radius $h$, a ripple period $p$, and a ripple amplitude $l$. Below, for the convenience of description, it will be referred to as the "coaxial electrostatic wiggler."

Compared to an electrostatic wiggler and an electromagnetic-wave wiggler, the advantage of a magnetic wiggler is related to practical feasibility of large magnetic field amplitudes. On the axis of a magnetic wiggler, as a matter of fact, the force acted by the magnetic wiggler field on an electron is $e v_{z} B \approx e c B\left(e, v_{z}, c\right.$, and $B$ being the electron's charge and axial velocity, the light speed in vacuum, and the amplitude of the magnetic wiggler, respectively), whereas the force produced by an electrostatic wiggler is $e E$. Therefore, relationship of the equivalence between the magnetic and electrostatic wigglers is $E \approx c B$; that is to say, the effect produced by a magnetic wiggler with field amplitude of $1 \mathrm{~T}$ is equivalent to that produced by an electrostatic wiggler with large field

\footnotetext{
*sczhang@home.swjtu.edu.cn
}

amplitude of $3 \times 10^{8} \mathrm{~V} / \mathrm{m}$. If an electromagnetic-wave wiggler is a planar wave, its power flow per unit area on cross section is $s=|\vec{E} \times \vec{H}|=c B^{2} / \mu_{0} \approx 2.387 \times$ $10^{14} B^{2}$. In other words, the effect produced by a magnetic wiggler with field amplitude of $1 \mathrm{~T}$ is equivalent to that produced by an electromagnetic-wave wiggler with large power flow of $2.387 \times 10^{14} \mathrm{~W} / \mathrm{m}^{2}$. It is due to this advantage that the magnetic wiggler is usually considered in FEL technology. Compared to an electromagnetic-wave wiggler and an electrostatic wiggler, however, a magnetic wiggler has the disadvantage that its period is restricted within the range of centimeters due to the state-of-art techniques, whereas the period of the electrostatic wiggler and the electromagnetic-wave wiggler can easily be extended to the range of millimeters. Therefore, attention has also been paid to the electrostatic and electromagnetic-wave wigglers.

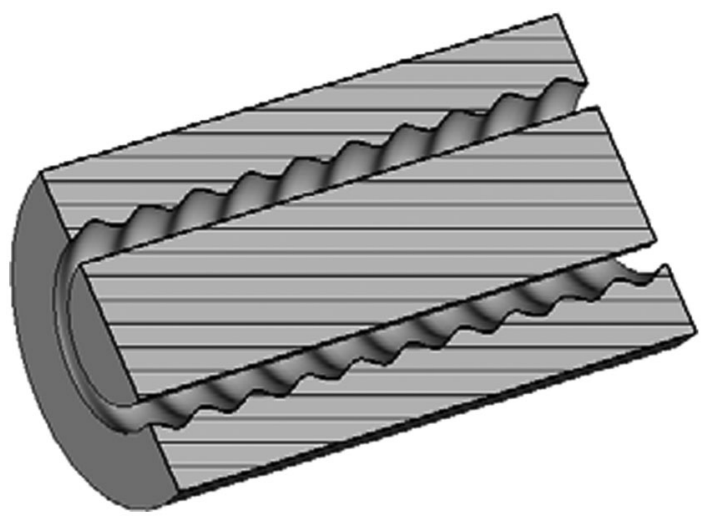

FIG. 1. Schematic diagram of a coaxial electrostatic wiggler, which is performed by a coaxial structure: the outside surface of the inner rod is smooth and grounded; the inside surface of the outer conductor is connected to a negative voltage and corrugated with sinusoidal ripples. 
Recently, a two-dimensional dusty plasma crystal [12] or a planar electrostatic system [13] was proposed as an electrostatic wiggler for FEL. Excluding the space-charge wave in the aforementioned three-wave interaction mechanism, it is found that a planar electrostatic wiggler [13] may be favorable for a mildly relativistic electron beam to generate terahertz waves by extracting the kinetic energy of the electrons [14]. Moreover, further study shows an interesting phenomenon that a coaxial electrostatic wiggler may pump both the kinetic energy and the electrostatic potential energy of a relativistic electron beam interacting with a transverse-electric wave, resulting in the wave amplification with ultrahigh gain [15].

Although the coaxial electrostatic wiggler was often used before, to the best of my knowledge, so far its physical and mathematical issues have not been rigorously treated. Since an explicit expression of the field distribution in a coaxial electrostatic wiggler is of significance in analyzing a coaxial-electrostatic-wiggler FEL, this paper is devoted to a rigorous treatment in mathematics and physics for this topic. The present paper is organized as follows. In Sec. II a theoretical model is described. The explicit expressions of the general solution and the special solution of the field distribution are mathematically derived in Secs. III and VI, respectively. Comparison between the explicit formulas and the software computer simulation technology (CST) [16] simulation results is given in Sec. V. Finally, conclusions and discussion are presented in Sec. VI.

\section{PHYSICAL AND MATHEMATICAL MODEL}

Adopting a cylindrical coordinate system $(r, \varphi, z)$ shown in Fig. 2, one can write the boundary function of the inside surface of the outer conductor as

$$
r_{\text {out }}=h-l \cos (k z)
$$

where $k=2 \pi / p$. Under the assumption that the transverse dimension is much smaller than the length in the $z$ direction, the electrostatic potential $\phi(r, z)$ in the physical system leads to the boundary value problem of the twodimensional Laplace equation,

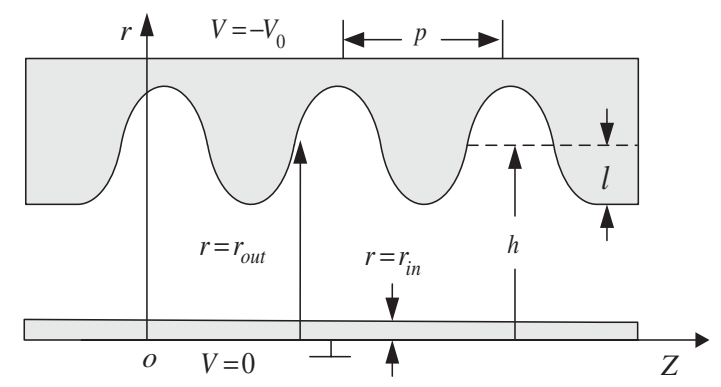

FIG. 2. Cylindrical coordinate system $(R, \varphi, z)$ adopted in the present paper.

$$
\frac{\partial^{2} \phi(r, z)}{\partial r^{2}}+\frac{\partial^{2} \phi(r, z)}{\partial z^{2}}=0
$$

with the boundary conditions

$$
\begin{gathered}
\left.\phi(r, z)\right|_{r_{\text {in }}=0}=0, \\
\left.\phi(r, z)\right|_{r=h-l \cos (k z)}=-V_{0},
\end{gathered}
$$

and the periodical condition

$$
\phi(r, z)=\phi(r, z+p) .
$$

In the following process, we derive the general solution by solving Eq. (2), and then determine the special solution by making use the boundary conditions and the periodical condition equations (3)-(5).

\section{DERIVATION OF GENERAL SOLUTION}

We employ the method of separation of variables by letting

$$
\phi(r, z)=R(r) Z(z)
$$

Substituting it into (2), one can obtain two ordinary differential equations:

$$
\begin{gathered}
\frac{1}{r} \frac{d}{d r}\left(r \frac{d R}{d r}\right)-\lambda R(r)=0, \\
\frac{d^{2} Z(z)}{d z^{2}}+\lambda Z(z)=0,
\end{gathered}
$$

where $\lambda$ is the separation constant.

We deal with Eqs. (7) and (8) in the following three aspects.

(i) The separation constant $\lambda$ is negative: $\lambda=-q^{2}<0$ ( $q$ being a nonzero real number). In this situation the solution of (8) is

$$
Z(z)=g_{1} e^{q z}+g_{2} e^{-q z},
$$

where $g_{1}$ and $g_{2}$ are the integral constants. To satisfy the periodic condition (5), obviously, both $g_{1}$ and $g_{2}$ must be zero, which leads to a trivial solution and should be abnegated.

(ii) The separation constant $\lambda$ is zero: $\lambda=0$. Then, the general solutions of (7) and (8) are as follows:

$$
\begin{gathered}
Z(z)=a_{0}{ }^{\prime} z+b_{0}{ }^{\prime}, \\
R(r)=c_{0}{ }^{\prime} \ln r+c_{1}{ }^{\prime},
\end{gathered}
$$

where $a_{0}{ }^{\prime}, b_{0}{ }^{\prime}, c_{0}{ }^{\prime}$, and $c_{1}{ }^{\prime}$ are the integral constants. In order to satisfy the periodic condition (5), one must let $a_{0}{ }^{\prime}=0$. When $\lambda=0$, therefore, the solution of the electrostatic potential is

$$
\phi_{0}(r, z)=d_{1} \ln r+d_{0},
$$


where $d_{1}=b_{0}{ }^{\prime} c_{0}{ }^{\prime}$ and $d_{0}=b_{0}{ }^{\prime} c_{1}{ }^{\prime}$ are the integral constants.

(iii) The separation constant $\lambda$ is positive: $\lambda=q^{2}>0(q$ being a nonzero real number). In this case the solution of $(8)$ is

$$
Z(z)=a \cos (q z)+b \sin (q z),
$$

where $a$ and $b$ are the integral constants. Inserting (13) into the periodic condition (5) yields

$$
\begin{aligned}
a \cos (q z)+b \sin (q z)= & a \cos (q z+q p) \\
& +b \sin (q z+q p),
\end{aligned}
$$

which leads to the eigenvalues

$$
q p=2 n \pi, \quad \text { i.e., } q=\frac{2 \pi n}{p}=k n, \quad n=1,2,3, \ldots,
$$

where $n=0$ which corresponds to $q=0$ and $\lambda=0$ has been excluded. Substituting $\lambda=q^{2}>0$ into (7) results in

$$
(q r)^{2} \frac{d^{2} R}{d r^{2}}+(q r) \frac{d R}{d r}-(q r)^{2} R=0 .
$$

Letting

$$
q r=t
$$

and inserting it into (16) yields

$$
t^{2} \frac{d^{2} R}{d t^{2}}+t \frac{d R}{d t}-\left(t^{2}-0\right) R=0 .
$$

Equation (18) is the first kind of modified Bessel equation of zero order with respect to the variable $t$. It has the following general solution:

$$
R(r)=c^{\prime}\left[I_{0}(q r)+\zeta^{\prime} K_{0}(q r)\right],
$$

where $c^{\prime}$ and $\zeta^{\prime}$ are the integral constants, $I_{0}$ and $K_{0}$ are the first and second kind of modified Bessel functions of zero order, respectively. Substituting the eigenvalues (15) into (13) and (19), we obtain

$$
\begin{array}{ll}
Z_{n}(z)=a_{n} \cos (n k z)+b_{n} \sin (n k z), & n=1,2,3, \ldots \\
R_{n}(r)=c_{n}\left[I_{0}(n k r)+\zeta_{n} K_{0}(n k r)\right], & n=1,2,3, \ldots
\end{array}
$$

Inserting (20) and (21) into (6), we find

$$
\begin{aligned}
\phi_{n}(r)= & c_{n}\left[I_{0}(n k r)+\zeta_{n} K_{0}(n k r)\right] \\
& \times\left[a_{n} \cos (n k z)+b_{n} \sin (n k z)\right] \quad(n=1,2,3, \ldots) .
\end{aligned}
$$

Superposing the solutions for both $\lambda=0$ and for $\lambda=$ $q^{2}>0$, Eqs. (12) and (22), we obtain the general solution of Eq. (2):

$$
\begin{aligned}
\phi(r, z)= & d_{0}+d_{1} \ln r+\sum_{n=1}^{\infty} c_{n}\left[I_{0}(n k r)+\zeta_{n} K_{0}(n k r)\right] \\
& \times\left[a_{n} \cos (n k z)+b_{n} \sin (n k z)\right] .
\end{aligned}
$$

Compared to the ordinary structure without ripples, obviously, the last term on the right-hand side of Eq. (23) represents the effect of the ripples on the field distribution.

\section{DETERMINATION OF SPECIAL SOLUTION}

Below, we determine the special solution by making use of the boundary conditions. Inserting (23) into the inner boundary condition (3) yields

$$
\begin{aligned}
d_{0} & +d_{1} \ln r_{\text {in }}+\sum_{n=1}^{\infty} c_{n}\left[I_{0}\left(n k r_{\text {in }}\right)+\zeta_{n} K_{0}\left(n k r_{\text {in }}\right)\right] \\
& \times\left[a_{n} \cos (n k z)+b_{n} \sin (n k z)\right]=0 .
\end{aligned}
$$

Balancing both sides of this equation requires

$$
d_{0}+d_{1} \ln r_{\text {in }}=0 \quad I_{0}\left(n k r_{\text {in }}\right)+\zeta_{n} K_{0}\left(n k r_{\text {in }}\right)=0 \text {, }
$$

which leads to

$$
d_{0}=-d_{1} \ln r_{\mathrm{in}}
$$

and

$$
\zeta_{n}=-\frac{I_{0}\left(n k r_{\text {in }}\right)}{K_{0}\left(n k r_{\text {in }}\right)}
$$

Consequently, (23) can be rewritten as

$$
\begin{aligned}
\phi(r, z)= & d_{1} \ln r-d_{1} \ln r_{\text {in }}+\sum_{n=1}^{\infty} c_{n}\left[I_{0}(n k r)+\zeta_{n} K_{0}(n k r)\right] \\
& \times\left[a_{n} \cos (n k z)+b_{n} \sin (n k z)\right],
\end{aligned}
$$

where $\zeta_{n}$ must be fixed by (27). Then, substituting (28) into the outer boundary condition (4) results in

$$
\begin{aligned}
-V_{0}= & d_{1} \ln r_{\text {out }}-d_{1} \ln r_{\text {in }}+\sum_{n=1}^{\infty} c_{n}\left[I_{0}\left(n k r_{\text {out }}\right)\right. \\
& \left.+\zeta_{n} K_{0}\left(n k r_{\text {out }}\right)\right]\left[a_{n} \cos (n k z)+b_{n} \sin (n k z)\right] .
\end{aligned}
$$

Noting that $r_{\text {out }}=h-l \cos (k z)$ is a function of the variable $z$, we rewrite the term $d_{1} \ln r_{\text {out }}$ in (29) as

$$
\begin{aligned}
d_{1} \ln r_{\text {out }} & =d_{1} \ln [h-l \cos (k z)] \\
& =d_{1} \ln h+d_{1} \ln \left[1-\frac{l}{h} \cos (k z)\right] .
\end{aligned}
$$

Suppose $h \gg l$ and $r_{\text {out }} \approx h$ so that we approximately get

$$
I_{0}\left(n k r_{\text {out }}\right)+\zeta_{n} K_{0}\left(n k r_{\text {out }}\right) \approx I_{0}(n k h)+\zeta_{n} K_{0}(n k h) .
$$

It will be shown in Sec. $\mathrm{V}$ that this approximation is quite reasonable. Substituting (30) and (31) into (29) yields 


$$
\begin{aligned}
-V_{0}= & -d_{1} \ln r_{\mathrm{in}}+d_{1} \ln h+d_{1} \ln \left[1-\frac{l}{h} \cos (k z)\right] \\
& +\sum_{n=1}^{\infty} c_{n}\left[I_{0}(n k h)+\zeta_{n} K_{0}(n k h)\right]\left[a_{n} \cos (n k z)\right. \\
& \left.+b_{n} \sin (n k z)\right],
\end{aligned}
$$

or

$$
\begin{aligned}
- & V_{0}-d_{1} \ln \left[1-\frac{l}{h} \cos (k z)\right] \\
= & {\left[-d_{1} \ln r_{\mathrm{in}}+d_{1} \ln h\right]+\sum_{n=1}^{\infty} c_{n}\left[I_{0}(n k h)+\zeta_{n} K_{0}(n k h)\right] } \\
& \times\left[a_{n} \cos (n k z)+b_{n} \sin (n k z)\right] .
\end{aligned}
$$

To balance both sides of this equation, one must let the constant term and the functional term to $z$ on the left-hand side be equal to the ones on the right-hand side:

$$
\begin{aligned}
-V_{0} & =d_{1} \ln \frac{h}{r_{\text {in }}}, \\
-d_{1} \ln \left[1-\frac{l}{h} \cos (k z)\right]= & \sum_{n=1}^{\infty} c_{n}\left[I_{0}(n k h)+\zeta_{n} K_{0}(n k h)\right] \\
& \times\left[a_{n} \cos (n k z)+b_{n} \sin (n k z)\right] .
\end{aligned}
$$

Equation (34) determines the integral constant

$$
d_{1}=-\frac{V_{0}}{\ln \left(h / r_{\text {in }}\right)} .
$$

In order to determine the other integral constants, we make use of the series $\ln (1 \mp u)=\mp u-\frac{1}{2} u^{2} \mp \frac{1}{3} u^{3}-$ $\frac{1}{4} u^{4} \mp \cdots$ and expand

$$
\begin{aligned}
\ln \left[1-\frac{l}{h} \cos (k z)\right]= & -\frac{l}{h} \cos (k z)-\frac{1}{2}\left(\frac{l}{h}\right)^{2} \cos ^{2}(k z) \\
& -\frac{1}{3}\left(\frac{l}{h}\right)^{3} \cos ^{3}(k z)-\cdots .
\end{aligned}
$$

Substituting (37) into (35) and balancing both sides, we obtain

$$
b_{n} \equiv 0
$$

and

$$
\begin{aligned}
& a_{n}=0, \quad n=2,3,4, \ldots \\
& c_{1} a_{1}=\frac{-V_{0}(l / h)}{\left[I_{0}(k h)+\zeta_{1} K_{0}(k h)\right] \ln \left(h / r_{\text {in }}\right)}, \quad n=1,
\end{aligned}
$$

where

$$
\zeta_{1}=-\frac{I_{0}\left(k r_{\text {in }}\right)}{K_{0}\left(k r_{\text {in }}\right)}
$$

Finally, substituting (36), (38), and (39) into (28), we obtain the special solution of the electrostatic potential:

$$
\begin{aligned}
\phi(r, z)= & -\frac{V_{0}}{\ln \left(h / r_{\text {in }}\right)} \ln \left(r / r_{\text {in }}\right) \\
& -\frac{V_{0}(l / h)}{\left[I_{0}(k h)+\zeta_{1} K_{0}(k h)\right] \ln \left(h / r_{\text {in }}\right)} \\
& \times\left[I_{0}(k r)+\zeta_{1} K_{0}(k r)\right] \cos (k z) .
\end{aligned}
$$

\section{COMPARISON OF ANALYTICAL SOLUTION WITH NONLINEAR SIMULATIONS}

One software often used in the electromagnetic computation is the CST [16], which is based on the nonlinear selfconsistent Maxwell's equations together with the boundary conditions. In this section we examine the special solution by comparing with the results of the CST simulations. By inserting (41) into the definition of electric field $\vec{E}=-\nabla \phi$, the $r-, \varphi$-, and $z$-direction components of the electric field can then be figured out:
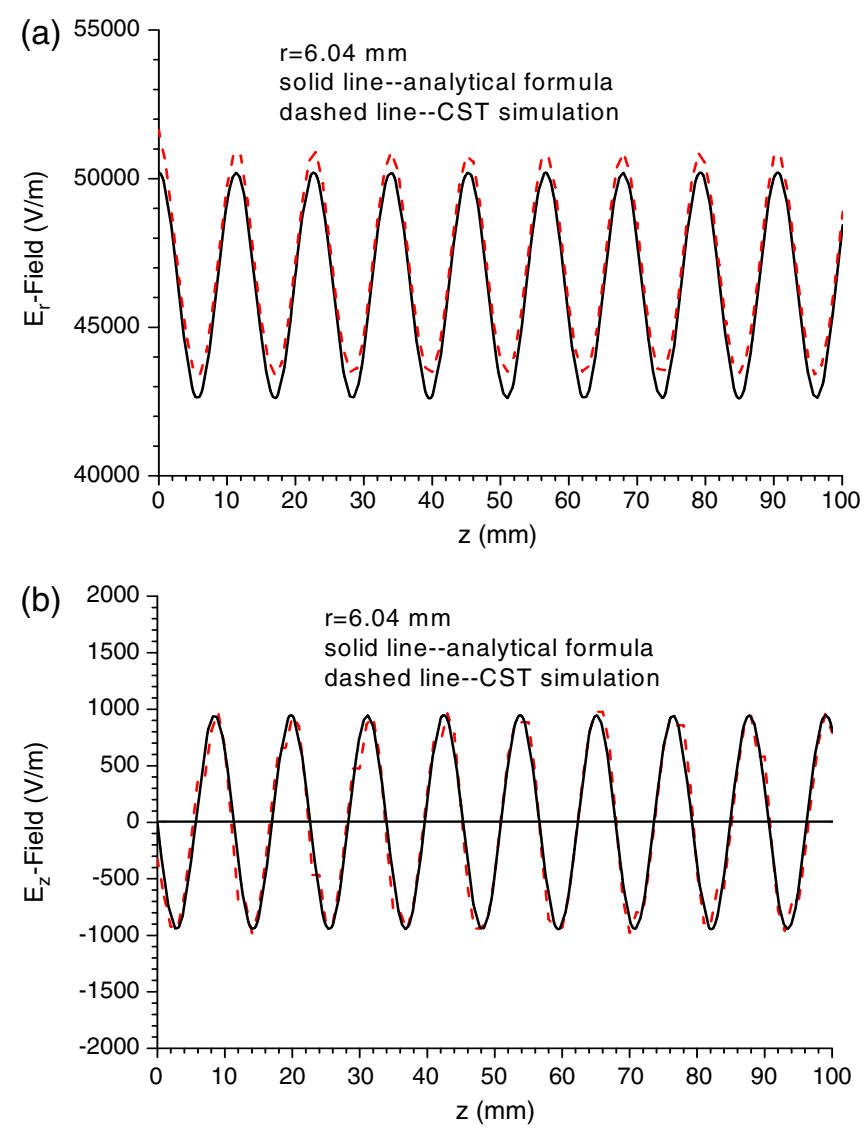

FIG. 3. Electric field components in the $r$ direction (a) and the $z$ direction (b) versus the longitudinal position $z$ at a location of $r=6.04 \mathrm{~mm}$, where $l=0.3 \mathrm{~mm}, h=8 \mathrm{~mm}, p=11.32 \mathrm{~mm}$, $r_{\text {in }}=5.6 \mathrm{~mm}$, and $V_{0}=100 \mathrm{~V}$. 


$$
\begin{aligned}
& E_{r}(r, z)=\frac{V_{0}}{\ln \left(h / r_{\text {in }}\right)} \frac{1}{r}+\frac{V_{0} k(l / h)}{\left[I_{0}(k h)+\zeta_{1} K_{0}(k h)\right] \ln \left(h / r_{\text {in }}\right)} \\
& \times\left[I_{1}(k r)+\zeta_{1} K_{1}(k r)\right] \cos (k z), \\
& E_{z}(r, z)=-\frac{V_{0} k(l / h)}{\left[I_{0}(k h)+\zeta_{1} K_{0}(k h)\right] \ln \left(h / r_{\mathrm{in}}\right)}\left[I_{1}(k r)\right. \\
& \left.+\zeta_{1} K_{1}(k r)\right] \sin (k z) \\
& E_{\varphi}(r, z)=0
\end{aligned}
$$

where $I_{1}$ and $K_{1}$ are the first and second kind of modified Bessel functions of order one, respectively. When $l=0$ (i.e., no corrugated ripples), all the expressions derived above go back to the well-known ones of a smooth system:
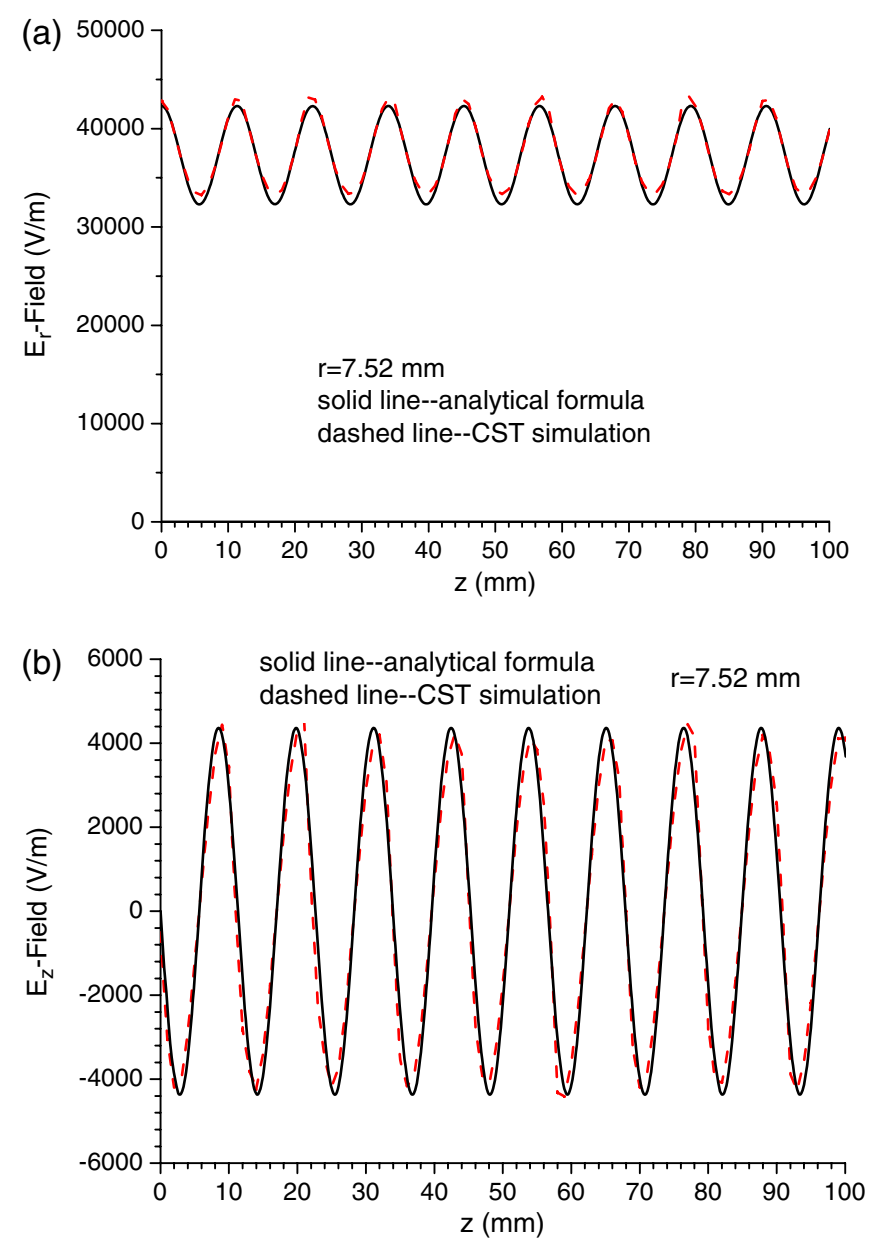

FIG. 4. Electric field components in the $r$ direction (a) and the $z$ direction (b) versus the longitudinal position $z$ at a location of $r=7.52 \mathrm{~mm}$, where the other parameters are the same as in Fig. 3.

$$
E_{r}(r, z)=\frac{V_{0}}{\ln \left(h / r_{\text {in }}\right)} \frac{1}{r}, \quad E_{\varphi}(r, z)=E_{z}(r, z)=0 .
$$

Physically speaking, therefore, expressions (42) and (43) conclude the effect of the corrugated ripples on the electric field.

Now we choose a typical set of parameters to compare the analytical formulas with CST simulation results: $l=0.3 \mathrm{~mm}, h=8 \mathrm{~mm}, p=11.32 \mathrm{~mm}, r_{\text {in }}=5.6 \mathrm{~mm}$, and $V_{0}=100 \mathrm{~V}$. Figures 3 and 4 provide comparison of the calculation results of the radial and longitudinal components of the electric field at location of $r=6.04 \mathrm{~mm}$ and $7.52 \mathrm{~mm}$, respectively, where the solid lines are obtained from the analytical expressions (42) and (43) derived in the present paper, and the dashed lines from the CST simulations. The azimuthal component simulated by the CST is zero, which exactly coincides with Eq. (44). We have noted that, in Figs. 3(a) and 4(a), there is a small systematic shift of the radial component calculated by the analytical formula and by the CST simulations. The reason may be the disappearance of the harmonic terms in Eq. (32) in the derivation process from Eq. (37) to (40). Nevertheless, excellent agreement between the analytical expressions and the CST simulation can be found in Figs. 3 and 4.

\section{CONCLUSIONS AND DISCUSSION}

A coaxial electrostatic wiggler leads to a twodimensional Laplace equation with a coaxial sinusoidal boundary value problem. By using separation of variables, both the general solution and the special solution have been mathematically derived, which are the sine or cosine functions with respect to the axial variable and are related to the first and second kind of modified Bessel functions to the radial variable, respectively. This distribution reflects the effect of the sinusoidal-ripple boundary on the electric field. Comparison has demonstrated excellent agreement between the analytical formulas and the CST simulations.

Since the field distribution shown by (42)-(44) is similar to those in a magnetic wiggler and a planar electrostatic wiggler, a coaxial electrostatic wiggler could be expected to modulate the motion of relativistic electrons, just as a magnetic wiggler or a planar electrostatic wiggler does $[13,17,18]$. Nonlinear study further confirms efficient amplification of an electromagnetic wave at a frequency of $120 \mathrm{GHz}$ with an output power of 2.7 megawatt (MW) and an ultrahigh gain of $74 \mathrm{~dB}$, where the coaxial electrostatic wiggler extracts both the kinetic energy and the electrostatic potential energy of the relativistic electron beam to the wave [15].

\section{ACKNOWLEDGMENTS}

The author thanks Y. Kong for assistance with the CST simulation in Figs. 3 and 4. This work is partly supported by the NSFC (No. 60871023) and the China UniversityCollege Ph.D. Science Foundation (No. 200806130012). 
[1] G. Bekefi and R. E. Shefer, J. Appl. Phys. 50, 5158 (1979).

[2] G. Bekifi, J. Appl. Phys. 51, 3081 (1980).

[3] A. Anselmo and J. A. Nation, IEEE Trans. Nucl. Sci. 32, 3494 (1985).

[4] A. Anselmo and J.A. Nation, Phys. Fluids 31, 2037 (1988).

[5] D. L. Fenstermacher and C. E. Seyler, Phys. Fluids 30, 190 (1987).

[6] S. Liu and X. Cheng, J. Appl. Phys. 62, 3104 (1987).

[7] P. Uddholm, J. E. Willett, and S. Bilikman, J. Phys. D 24, 1278 (1991).

[8] J. E. Willett, Y. Aktas, B. Maraghechi, and H. Mehdian, J. Phys. D 27, 211 (1994).

[9] H. Mehdian, J. E. Willett, H. Mehdian, and Y. Aktas, Phys. Plasmas 3, 1130 (1996).
[10] S. Riyopoulos, Phys. Rev. E 56, 4710 (1997).

[11] B. Maraghechi and N.S. Javan, Phys. Plasmas 8, 4193 (2001).

[12] S. Mirzanejhad and B. Bahadory, Phys. Plasmas 14, 043101 (2007).

[13] S.-C. Zhang, J. Infrared, Millimeter, and Terahertz Waves 31, 249 (2010).

[14] S.-C. Zhang, Phys. Plasmas 16, 093107 (2009).

[15] S.-C. Zhang, Phys. Plasmas 17, 053102 (2010).

[16] Computer Simulation Technology (CST), User's Manual 5, in CST-Microwave Studio, 2003.

[17] J. M. J. Madey, J. Appl. Phys. 42, 1906 (1971).

[18] L. R. Elias, W. M. Fairbank, J. M. J. Madey, H. A. Schwettman, and T. I. Smith, Phys. Rev. Lett. 36, 717 (1976). 